\title{
Advances to improve the eating and cooking qualities of rice by marker-assisted breeding
}

\begin{abstract}
The eating and cooking qualities of rice are heavily emphasized in breeding programs because they determine market values and they are the appealing attributes sought by consumers. Conventional breeding has developed traditional varieties with improved eating and cooking qualities. Recently, intensive genetic studies have pinpointed the genes that control eating and cooking quality traits. Advances in genetic studies have developed molecular techniques, thereby allowing marker-assisted breeding (MAB) for improved eating and cooking qualities in rice. $\mathrm{MAB}$ has gained the attention of rice breeders for the advantages it can offer that conventional breeding cannot. There have been successful cases of using $\mathrm{MAB}$ to improve the eating and cooking qualities in rice over the years. Nevertheless, MAB should be applied cautiously given the intensive effort needed for genotyping. Perspectives from conventional breeding to marker-assisted breeding will be discussed in this review for the advancement of the eating and cooking qualities of fragrance, amylose content (AC), gel consistency (GC) and gelatinization temperature (GT) in rice. These four parameters are associated with eating and cooking qualities in rice. The genetic basis of these four parameters is also included in this review. MAB is another approach to rice variety improvement and development in addition to being an alternative to genetic engineering. The MAB approach shortens the varietal development time, and is therefore able to deliver improved rice varieties to farmers within a shorter period of time.
\end{abstract}

Keyword: Amylose content; Conventional breeding; Fragrance; Gel consistency; Gelatinization temperature; Marker-assisted backcross breeding; Marker-assisted selection; Quantitative trait loci 